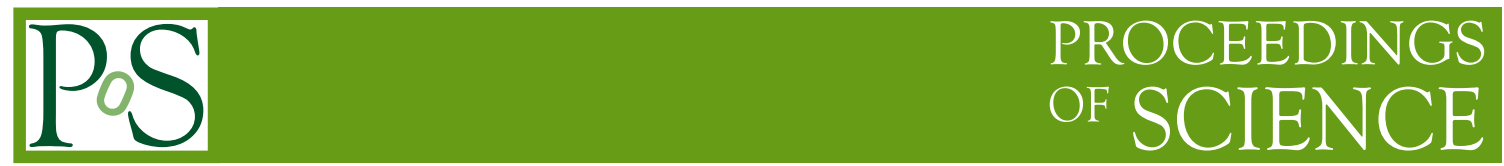

\title{
Operations and Data Taking Status of ADMX
}

\section{Tatsumi Nitta for the ADMX collaboration}

University of Washington, Seattle, Washington 98195, USA

E-mail: tnitta1@uw.edu

The axion is a hypothetical particle arising from the Peccei-Quinn solution to the strong- $C P$ roblem, and an excellent candidate for dark matter. The Axion Dark Matter Experiment (ADMX) is an experiment that searches for axions as a dark matter with a resonant cavity under a strong superconducting magnetic field. In previous operations, ADMX achieved sensitivity to the GUTinspired DFSZ axion model between 2.7-3.3 $\mu \mathrm{eV}$ with yocto Watt level background using a quantum amplifier and dilution refrigerator. The latest run has been in data-taking since 2020. In this run, we have improved our blind axion signal injection, improved our operating efficiency, and have new methods to distinguish true axion signals from the background. I will discuss these advances as well as the current data-taking status.

\footnotetext{
*** The European Physical Society Conference on High Energy Physics (EPS-HEP2021), *** *** 26-30 July $2021 * * *$

*** Online conference, jointly organized by Universität Hamburg and the research center DESY ***
} 
The axion is a hypothetical particle introduced to solve the strong- $C P$ problem [1-3], and one of the most promising candidates of dark matter [4-7]. The axion emerges as a Nambu-Goldstone boson by cause of the global U(1) axial symmetry (Peccei-Quinn symmetry) breaking at the early universe. Depends on the symmetry breaking scale, there are several mass predictions, though most of the mass predictions lie between $O(1-100) \mu \mathrm{eV}$ [8-19]. The axion has a coupling to two photons, and the numerical values are represented by two benchmarks, the Kim-Shifman-VainshteinZakharov (KSVZ) [20,21] and Dine-Fischler-Srednicki-Zhitnitsky (DFSZ) [22, 23] models. Due to the axion-photon coupling, the existence of axion fields modifies Maxwell's Equations and produces a effective current, $\mathbf{J}_{\mathrm{eff}}=g_{a \gamma \gamma} \mathbf{B} \partial_{t} a$, where $\mathbf{B}$ is the magnetic flux density, $g_{a \gamma \gamma}$ is the axion-photon coupling constant, and $a$ is the axion field. The frequency of the effective current matches to $E_{a} / h \sim m_{a} / h$, where $h$ is the Planck's constant, $E_{a}$ and $m_{a}$ are energy and mass of the axion.

Several direct detection experiments with the axion-photon coupling and a resonant cavity have succeeded in searching for the axion dark matter, such as HAYSTAC [24, 25] and experiments at CAPP [26-28]. Axion Dark Matter eXperiment (ADMX) is the first and only experiment that succeeded in searching for the DFSZ axion dark matter [29-34]. The ADMX apparatus consists of a resonant cavity immersed in a strong magnetic field, where axion fields are converted to photons and enhanced subsequently, and an ultra low noise RF receiver chain, which is realized by a Josephson Parametric Amplifier (JPA) [35] and a dilution refrigerator to minimize both the black body noise and electronic noise from the amplifier.

The previous operations of ADMX excluded DFSZ axions in the 2.7-3.3 $\mu \mathrm{eV}$ mass range. The latest operation has been performed from October 2019 to May 2021, which searched for axions in the 3.1-4.2 $\mu \mathrm{eV}$ mass range. Compared to the previous operation [34], we have implemented several improvements as follows. We introduced scanning with an overcoupling state to increase scan speed. The scan speed is proportion to $\beta^{2} /(1+\beta)^{3}$ where $\beta=Q_{0} / Q_{e}, Q_{0}$ and $Q_{e}$ is unloaded and external quality factors for a cavity because overcoupling state has a wider enhancement of the cavity across digitization range. Hence, $\beta=2$ is the fastest, corresponds to a $20 \%$ improvement in the scan speed. The second scan, the scan for significant excesses identified from the first scan, was performed with $\beta=1$ because those excesses have a narrow width compared to the quality factor of the cavity. Another improvement was an optimization of the JPA rebiasing algorithm. In the previous operations, we have performed fine-tuning the bias current and pump power of the JPA to maximize the JPA performance every four digitizations, which takes around 100 seconds. However, those optimal values did not drastically change within nearby frequencies. Thus most of the optimization is unnecessary. Thereby, we introduced the algorithm that performing rebiasing only if the performance decreased. This improvement made a $20 \%$ improvement in the scan speed.

The Synthetic Axion Generator (SAG) signals, artificial signals having Maxwell-Boltzmann distribution produced by a waveform generator, were injected across the latest operation. A few SAG operators chose frequencies and the powers, and an independent operation team detected the SAGs to ensure our axion detection ability. An example of a SAG signal is shown in Figure 1. The left plot shows the detected SAG line shapes with $\mathrm{TM}_{010}$ (black) and $\mathrm{TM}_{011}$ (red) modes, respectively. The signal was always detected at the same frequency and enhanced at the center of the resonant frequency. This implies that the signal was in the cavity. The two plots on the right-hand side show that the impressed magnetic field and the electric fields for $\mathrm{TM}_{010}$ (left) and $\mathrm{TM}_{011}$ (right) modes, respectively. One can see that the $\mathrm{TM}_{011}$ has the opposite phase of the electric 

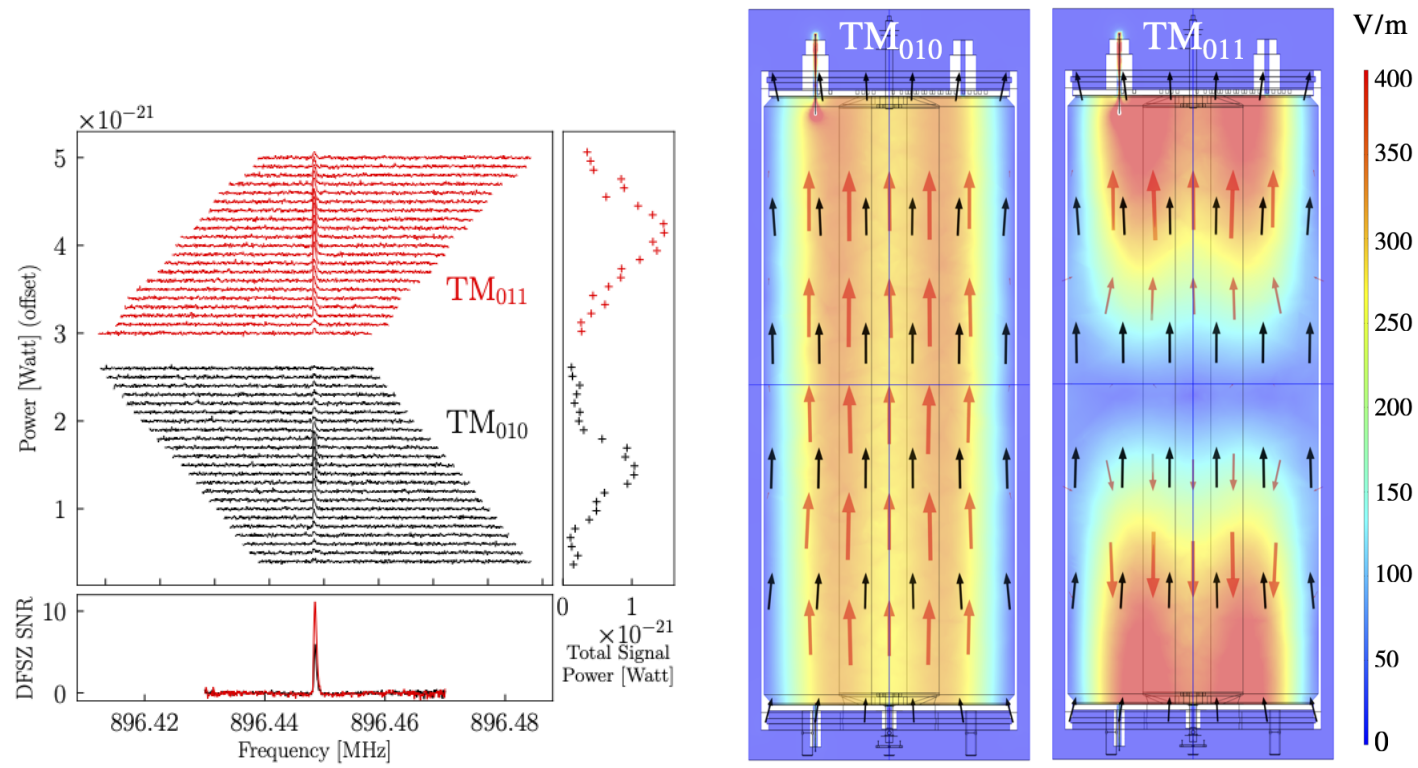

Figure 1: (left) Digitization spectra having a $\mathrm{SAG}$ signal for $\mathrm{TM}_{010}$ (black) and $\mathrm{TM}_{011}$ (red) modes. The bottom small plot shows signal to noise ratio (SNR) for the frequencies. The right small plots shows the total signal power in Watt over 30 bins ahead from the candidate frequency. (right) Electric field distribution for $\mathrm{TM}_{010}$ (left) and $\mathrm{TM}_{011}$ (right) modes evaluated with COMSOL Multiphysics [36]. Electric and magnetic fields are shown as red and black arrows, respectively. The overlap between the $\mathrm{TM}_{010}$ electric and magnetic field is large and consistent across the volume, while the overlap between the $\mathrm{TM}_{011}$ electric field is of opposite sign in the top and bottom of the cavity, leading to destructive interference.

field at the top and bottom of the cavity. Thus, induced effective currents by the axion would be destructively interfered, i.e., axion signal would not appeared in the $\mathrm{TM}_{011}$. However, we clearly observed the signal at the $\mathrm{TM}_{011}$ in the Figure 2. Therefore, we identified the candidate shown in Figure 1 is not the axion by this behavior.

From the latest operation, no axion-like candidate was found. Therefore, we set $90 \%$ confidence limits on the axion-photon coupling as shown in Figure 2. The red-colored and lighter red-colored areas show the limits assuming a boosted Maxwell-Boltzmann distribution [37] with $0.45 \mathrm{GeV} / \mathrm{cc}$ of the dark matter density and a result from N-body simulation with $0.6 \mathrm{GeV} / \mathrm{cc}$ of the dark matter density [38] as the velocity distribution for dark matter axions, respectively. We exclude KSVZ (DFSZ) axions in the mass range 3.3-4.2 (3.9-4.1) $\mu \mathrm{eV}$. The limit obtained from this work is the most stringent to date. Figure 3 shows experimental limits for the axion-photon couplings from other experiments and theory prediction. The limit from this work starts to exclude one of the promising models.

We plan to implement several improvements to scan over the same frequency with the complete DFSZ sensitivity. For instance, we will replace the cavity support from stainless steel with carbon fiber to mitigate heat flow to the cavity and replace the $\mu$-metal shield surrounding the JPA to decrease the leakage of the magnetic field. Furthermore, we plan to search the DFSZ dark matter axion with 4- and 18-cavities array to increase cavity resonant frequency. 


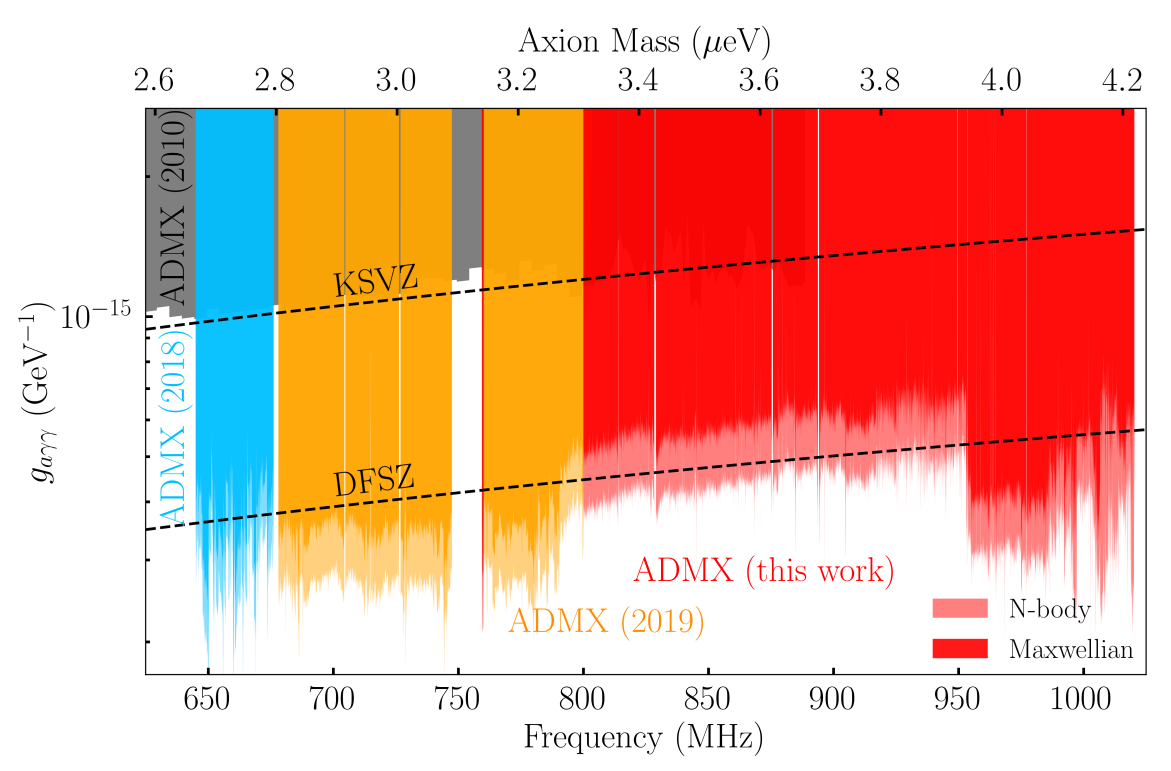

Figure 2: $90 \%$ C.L. on the $g_{a \gamma \gamma}$ for axion frequency. The gray-, blue-, and yellow-colored areas represent previously reported ADMX limits in References [29], [33], and [34]. The red-colored area is the limits from this work. We ruled out KSVZ (DFSZ) axions in the mass range 3.3-4.2 (3.9-4.1) $\mu \mathrm{eV}$.

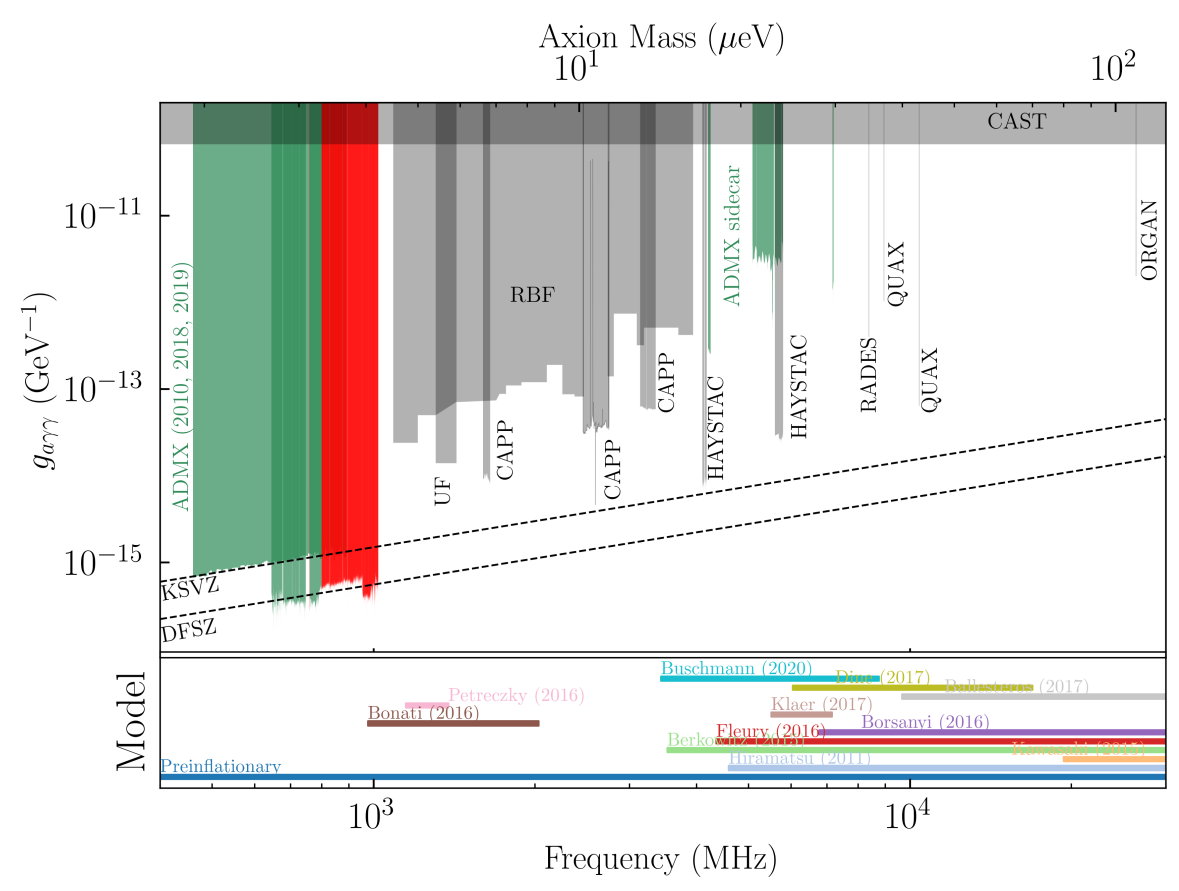

Figure 3: $90 \%$ C.L. on the $g_{a \gamma \gamma}$ for axion frequency. The green-colored areas represent previously reported ADMX limits in References [29], [33], and [34]. The red-colored area is the limits from this work. The gray-colored area is the limit from the other experiments [24-28, 39-45]. The Bottom small window shows the QCD analytical and numerical calculations from [8-19]. 


\section{References}

[1] R. D. Peccei and Helen R. Quinn. Phys. Rev. Lett., 38:1440-1443, Jun 1977.

[2] Steven Weinberg. Phys. Rev. Lett., 40:223-226, 1978.

[3] Frank Wilczek. Phys. Rev. Lett., 40:279-282, 1978.

[4] John Preskill, Mark B. Wise, and Frank Wilczek. Physics Letters B, 120(1):127-132, 1983.

[5] L.F. Abbott and P. Sikivie. Physics Letters B, 120(1):133-136, 1983.

[6] Michael Dine and Willy Fischler. Physics Letters B, 120(1):137-141, 1983.

[7] J. Ipser and P. Sikivie. Phys. Rev. Lett., 50:925-927, Mar 1983.

[8] S. Borsanyi et al. Nature, 539(7627):69-71, Nov 2016.

[9] Takashi Hiramatsu et al. Phys. Rev. D, 83:123531, Jun 2011.

[10] Masahiro Kawasaki, Ken'ichi Saikawa, and Toyokazu Sekiguchi. Phys. Rev. D, 91:065014, Mar 2015.

[11] Evan Berkowitz, Michael I. Buchoff, and Enrico Rinaldi. Phys. Rev. D, 92:034507, Aug 2015.

[12] Leesa Fleury and Guy D. Moore. Journal of Cosmology and Astroparticle Physics, 2016(01):004-004, jan 2016.

[13] Bonati et al. Journal of High Energy Physics, 2016(3):155, Mar 2016.

[14] Peter Petreczky, Hans-Peter Schadler, and Sayantan Sharma. Physics Letters B, 762:498-505, 2016.

[15] Guillermo Ballesteros et al. Phys. Rev. Lett., 118:071802, Feb 2017.

[16] Vincent B. Klaer and Guy D. Moore. Journal of Cosmology and Astroparticle Physics, 2017(11):049-049, nov 2017.

[17] Michael Dine et al. Phys. Rev. D, 96:095001, Nov 2017.

[18] Malte Buschmann et al. Phys. Rev. Lett., 124:161103, Apr 2020.

[19] Marco Gorghetto, Edward Hardy, and Giovanni Villadoro. SciPost Phys., 10:50, 2021.

[20] Jihn E. Kim. Phys. Rev. Lett., 43:103, 1979.

[21] Mikhail A. Shifman, A.I. Vainshtein, and Valentin I. Zakharov. Nucl.Phys., B166:493, 1980.

[22] Michael Dine, Willy Fischler, and Mark Srednicki. Phys. Lett., B104:199, 1981.

[23] A.R. Zhitnitsky. Sov. J. Nucl. Phys., 31:260, 1980. 
[24] B. M. Brubaker et al. Phys. Rev. Lett., 118:061302, Feb 2017.

[25] K. M. Backes et al. Nature, 590(7845):238-242, Feb 2021.

[26] S. Lee et al. Phys. Rev. Lett., 124:101802, Mar 2020.

[27] Junu Jeong et al. Phys. Rev. Lett., 125:221302, Nov 2020.

[28] Ohjoon Kwon et al. Phys. Rev. Lett., 126:191802, May 2021.

[29] S. Asztalos et al. Phys. Rev. D, 64:092003, Oct 2001.

[30] S. J. Asztalos et al. Phys. Rev. D, 69:011101, Jan 2004.

[31] S. J. Asztalos andothers. 571(1):L27-L30, may 2002.

[32] S. J. andothers Asztalos. Phys. Rev. Lett., 104:041301, Jan 2010.

[33] N. Du et al. Phys. Rev. Lett., 120:151301, Apr 2018.

[34] T. Braine et al. Phys. Rev. Lett., 124:101303, Mar 2020.

[35] I. Siddiqi, R. Vijay, F. Pierre, C. M. Wilson, M. Metcalfe, C. Rigetti, L. Frunzio, and M. H. Devoret. Physical Review Letters, 93(20), Nov 2004.

[36] COMSOL AB. Www. comsol. com.

[37] Michael S. Turner. Phys. Rev. D, 42:3572-3575, Nov 1990.

[38] Erik W. Lentz, Thomas R. Quinn, Leslie J Rosenberg, and Michael J. Tremmel. Astrophys. J., 845(2):121, 2017.

[39] W. U. Wuensch et al. Phys. Rev. D, 40:3153-3167, Nov 1989.

[40] C. Hagmann et al. Phys. Rev. D, 42:1297-1300, Aug 1990.

[41] D. Alesini et al. Phys. Rev. D, 99:101101, May 2019.

[42] D. Alesini et al. Phys. Rev. D, 103:102004, May 2021.

[43] A. Álvarez Melcón et al. 42021.

[44] Ben T. McAllister et al. Physics of the Dark Universe, 18:67-72, 2017.

[45] V. Anastassopoulos et al. Nature Physics, 13(6):584-590, Jun 2017. 